\title{
FECALOMA GIGANTE COMO CAUSA DE INCONTINENCIA E INESTABILIDAD VESICAL FEMENINA. APORTACIÓN DE UN CASO
}

\author{
M. ADRIAZOLA SEMINO, R. ORTIZ CABRIA, A. ALONSO VILLALBA, E. GARCÍA COBO, \\ E. TEJEDA, F. ROMERO
}

Servicio de Urología. Hospital Río Carrión. Palencia.

Actas Urol Esp. 28 (7): 527-529, 2004

RESUMEN

FECALOMA GIGANTE COMO CAUSA DE INCONTINENCIA E INESTABILIDAD VESICAL FEMENINA. APORTACIÓN DE UN CASO

Presentamos un caso de una paciente de 60 años con antecedentes de trastornos depresivos, intento de suicidio con secuelas de lesiones pélvicas, que acude al presentar incontinencia de urgencia e infecciones urinarias de repetición, de dos años de evolución. Es diagnosticada de gran fecaloma impactado que ocupa prácticamente el área pélvica, provocando desplazamiento de la vejiga y ectasia ureteral derecha.

PALABRAS CLAVE: Incontinencia. Fecaloma. Inestabilidad vesical.

\section{ABSTRACT}

GIANT FECAL IMPACTION AS A CAUSE OF FEMALE INCONTINENCE (FECALOMA) AND BLADDER INSTABILITY. CONTRIBUTION WITH ONE CASE

We present a case of a 60 years old female patient, with previous depressive disorders, an attempted suicide with pelvic injuries, who comes showing two years evolution of emergence incontinence. The was diagnosed with a giant fecal impaction occuping almost all the pelvic zone and leading to a bladder displacement and right ureteral ectasis.

PALABRAS CLAVE: Incontinence. Fecal impaction (Fecaloma). Bladder instability.

$\mathrm{L}$ as causas de incontinencia en la población geriátrica femenina son múltiples, entre ellas el normal envejecimiento fisiológico que conlleva una serie de cambios en las distintas estructuras del tracto urinario inferior (vesical y uretral), a consecuencia de una disminución de la tasa estrogénica que se produce en el climaterio.

Así mismo las alteraciones en el suelo pélvico (cistocele, rectocele), por una reducción de la relación fibra muscular/tejido conectivo.

Finalmente, existen una serie de factores que ayudan a potenciar la incontinencia como son: las infecciones urinarias, ingesta desmesurada de líquidos, diuréticos y falta de movilidad.

\section{CASO CLÍNICO}

Paciente de 60 años con antecedentes de trastornos depresivos, con intento de autolesión, provocándose politraumatismo con fractura de sacro, rama iliopubiana derecha (2 años de evolución); cursando posteriormente con infecciones urinarias de repetición, estreñimiento e incontinencia de patrón irritativo. 
A la exploración física presenta genitales externos atrógicos, test Boney negativo y discreto cistocele.

En la analítica presenta una bioquímica con parámetros normales y función renal conservada. Destaca un sedimento con leucocituria y bactiuria. El urocultivo está contaminado.

En el estudio urográfico presenta una buena función bilateral, con una moderada ectasia pieloureteral derecha, falta de repleción vesical y rechazo de la misma (Fig. 1).

Scan abdomino-pélvico: bazo, área pancreática, riñones con discreta ectasia ureteral derecha. Fecaloma gigante que ocupa la práctica totalidad de la pelvis y que produce gran desplazamiento vesical (Figs. 2 y 3).

Se consulta con la unidad de Digestivo, donde se procede a ingreso para extracción manual y terapia de enemas evacuantes; proceder a la limpieza del recto-sigma.

En posteriores controles se ha notado mejoría de su cuadro miccional y control del estreñimiento.

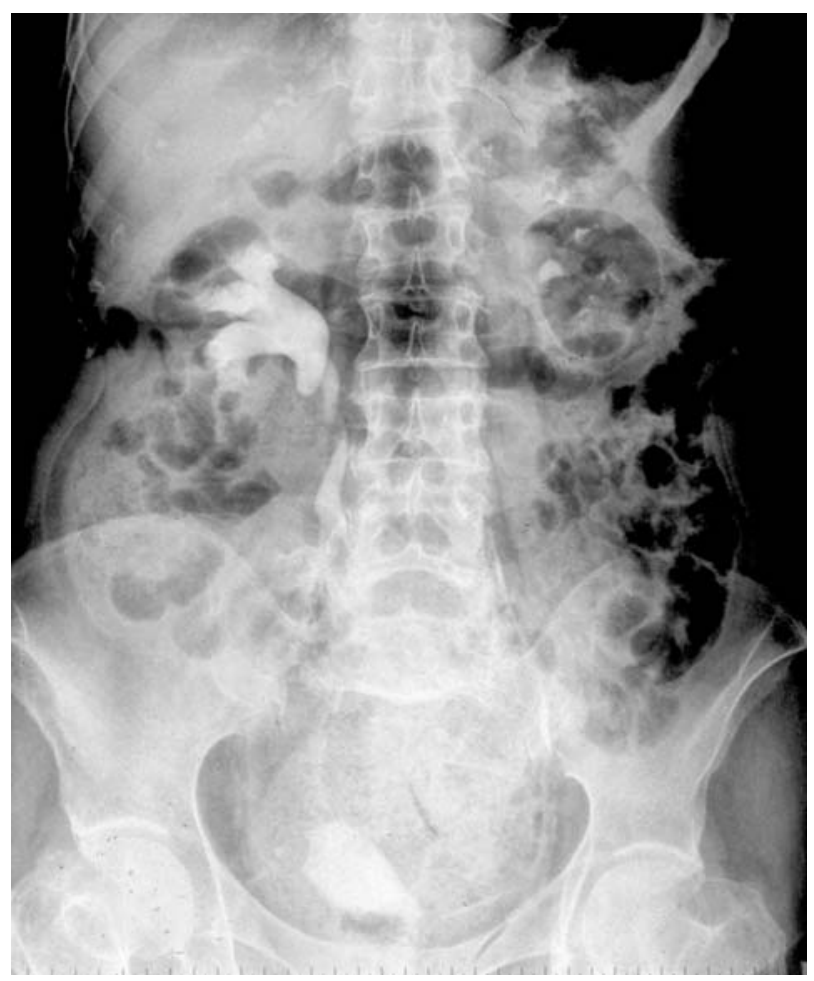

FIGURA 1. Urografias: se aprecia una buena eliminación de contraste bilateral, con una moderada ectasia pielocalicial izda. Desplazamiento vesical.

\section{DISCUSIÓN}

Al margen de los cambios orgánicos que aparecen en las mujeres mayores después del climaterio, existen causas transitorias de incontinencia en los ancianos y éstas básicamente aparecen reflejadas en la palabra anglo-sajona DIAPPERS, que de forma muy didáctica resume las causas de incontinencia en los pacientes geriátricos:

D: se trata del delirium, caracterizados por estados de confusión que pueden resultar por fallo cardiaco congestivo, trombosis venosa profunda, inclusive infecciones crónicas.

I: las infecciones del tracto urinario sintomático puede ser una causa de incontinencia y de inestabilidad vesical.

A: la vaginitis atrófica es una fuente frecuente de síntomas urinarios en los pacientes geriátricos, en el $80 \%$ de las mujeres con incontinencia presentan evidencia física de vaginitis atrofia.

P: existen diversos productos farmacológicos que están implicados en la incontinencia urinaria del anciano, entre ellos los diuréticos de asa, drogas hipnóticas, opiáceos, antihistamínicos, antidepresivos, antiparkinsonianos y los bloqueadores de calcio que están directamente asociados con estreñimiento y retención de orina.

P: trastornos psiquiátricos como ansiedad y depresión, conducen a este tipo de pacientes a cuadros de fugas miccionales.

E: el exceso de ingesta de líquidos en pacientes mayores, asociados con falta de movilidad, trastornos psico-motores, pueden conducir a inestabilidad y fuga. El exceso de diuresis contribuye a producir la incontinencia si ésta se asocia a nicturia.

$\mathrm{R}$ : la restricción de la movilidad por problemas osteomusculares, induce a falta de control miccional y fuga.

S: la inpactación fecal puede estar relacionada con la incontinencia en pacientes mayores en un $10 \%$ de los casos. Los pacientes presentan una incontinencia por imperiosidad o por rebosamiento y sufren también en algunos casos de incontinencia fecal. La desimpactación restablece la continencia.

Con independencia de la edad, movilidad, estado mental, la incontinencia no es nunca normal. Al atenuar la reserva fisiológica, el envejeci- 


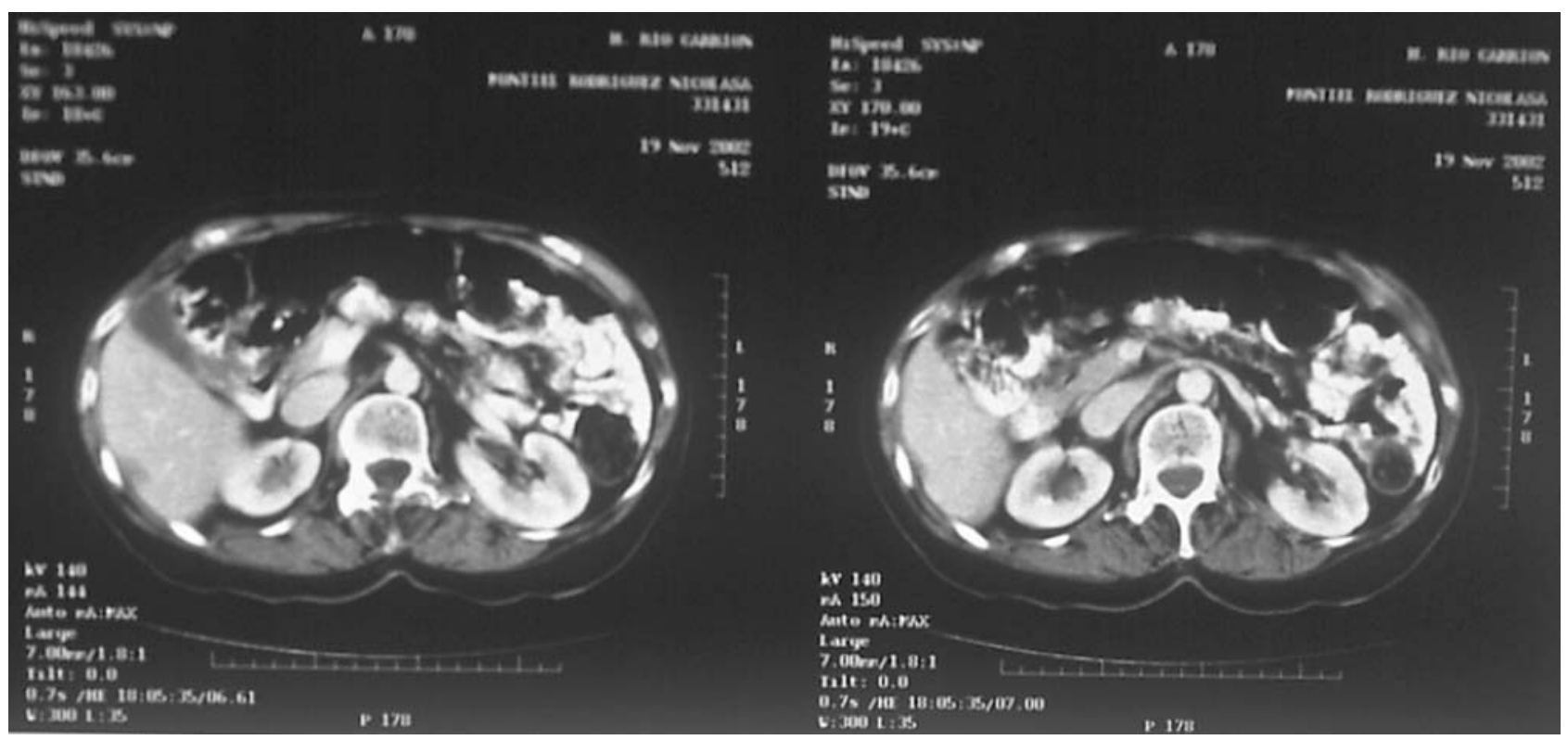

FIGURA 2. Scann abdomino-pélvico: con discreta uropatía obstructiva izda.

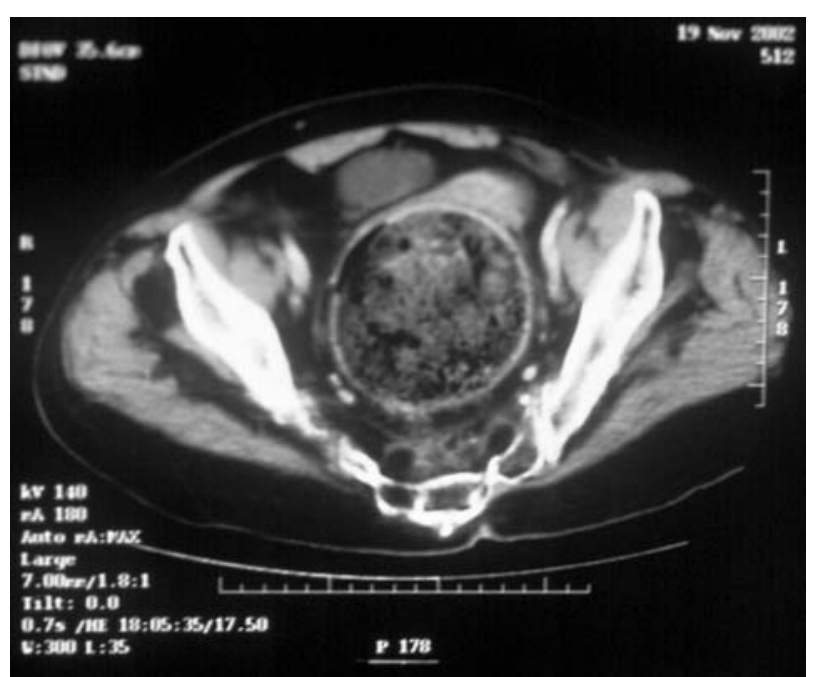

FIGURA 3. Scann pélvico donde se aprecia fecaloma gigante a nivel de recto-sigma.

miento aumenta la probabilidad de aparición de una incontinencia en presencia de una agresión fisiológica, farmacológica o patología asociada.

Presentamos un caso donde se juntan alteraciones orgánicas propias de la edad con factores externos como drogas, alteración psico-mental, falta de movilidad para producir inicialmente un cambio importante en el tránsito intestinal y finalmente la clínica urinaria.

\section{REFERENCIAS}

1. BORRIE MJ, CAMPELL K, ARCESE ZA: Urinary retention in patients in a geriatric rehabilitation. Unit Rehabilit Nurs 2001; 26 (5): 187-189.

2. BOUFFIOUX CH, LEVAL J.: La retention vesicale manifestation d una constipation opiniatre. Actas Urologica Belga 1975; 43: 77-83.

3. RESNICK NEIL.: Incontinencia geriátrica. Clínicas Urológicas Americanas 1996; 1: 61-82.

4. NELSON RP, BRUGH R et al.: Bilateral ureteral obstruction secondary to massive fecal impaction. Urology 1980 oct; 16: 403-406.

5. KANETTI J, BAR ZIV J.: Urinary retention due to fecal impaction in a child. Urology 1984 marzo; 23: 307.

6. DORAN J, ROBERTS M.: Acute retention in the female. British J Urology 1975; 47: 793-796.

7. DOUGHERTY MC, DYER JW, PENDERGAST JF.: A randomized trial of behavioural management for continence with older rural women. Research in Nursing/Health 2002 febrero; 25: 3-13.

Dr. M.A. Adriazola Semino

(Servicio de Urología)

Hospital Rio Carrión

Avda. Ponce de León, s/n

34005 Palencia

(Trabajo recibido el 10 junio de 2003) 